\title{
Daño neurológico secundario a malposición de catéter venoso central percutáneo en plexos paravertebrales
}

\author{
Neurological damage secondary to percutaneous central venous catheter \\ malposition in paravertebral plexuses
}

\author{
Nicksa Ubilla-Badilla', Manuel Montaña-Gutiérrez¹, Paulina Vargas-Mijalic ${ }^{1}$ \\ ${ }^{1}$ Matrona(ón) Unidad de Paciente Crítico Neonatal, Hospital Clínico Magallanes, Punta Arenas, Chile.
}

*Autor para correspondencia: nicksa.alibeck@gmail.com

RECIBIDO: 03 de Julio de 2020

APROBADO: 02 de Abril de 2021

(c) (P)

DOI: 10.22370/rev.mat.1.2021.2340

LOS AUTORES DECLARAN NO TENER CONFLICTO DE INTERESES

Palabras claves: Neonato, vena lumbar ascendente, nutrición parenteral, catéter venoso central percutáneo.

Key words: Ascendant lumbar vein, parenteral nutrition, percutaneous central venous catheter.

\section{RESUMEN}

Introducción y objetivo: Las complicaciones asociadas a la instalación de catéteres venosos centrales de inserción percutánea (PICC) son poco frecuentes y en ocasiones mortales. En el estudio, se describe el caso de un recién nacido prematuro que desarrolló daño neurológico secundario a la malposición de un PICC en plexos paravertebrales.

Identificar el mecanismo etiológico de la malposición de un PICC.

Metodología: Los datos del caso se recabaron del Hospital Clínico Magallanes, respetando la confidencialidad.

El manejo del caso se contrarresta con la evidencia encontrada en buscadores como National Center for Biotechnology Information, U.S. National Library of Medicine y Scientific Electronic Library Online.

Discusión: Las complicaciones asociadas a la malposición son infrecuentes en la literatura, sin embargo, se reportó el fallecimiento de un infante que previo a su muerte presentó síntomas neurológicos e inespecíficos.
Informe final y conclusiones: La malposición del PICC se asocia principalmente a la anatomía venosa de la extremidad inferior izquierda, esto propicia la malposición del catéter en plexos paravertebrales. He aquí la importancia como profesionales realizar una supervisión, control, pesquisa y derivación oportuna de cualquier signo de alteración en cada uno de nuestros pacientes.

\section{ABSTRACT}

Introduction and objective: Complications associated with the installation of central venous catheters for percutaneous insertion (PICC) are infrequent and sometimes fatal. The study describes the case of a premature newborn who developed neurological damage secondary to the malposition of a PICC in paravertebral plexuses.

Identify the etiological mechanism of the malposition of a PICC. 
Methodology: The data of the case were collected from the Hospital Clinico Magallanes, respecting confidentiality.

Case management is countered by evidence found in search engines such as the National Center for Biotechnology Information, U.S. National Library of Medicine and Scientific Electronic Library Online.

Discussion: Complications associated with malposition are infrequent in the literature, however, the death of an infant was reported who had neurological and nonspecific symptoms prior to death.

Final report and conclusions: The malposition of the PICC is mainly associated with the venous anatomy of the left lower extremity, this leads to the malposition of the catheter in paravertebral plexuses. This is the importance as professionals to carry out supervision, control, investigation and timely referral of any signs of alteration in each of our patients.

\section{INTRODUCCIÓN Y OBJETIVO}

Actualmente, existe controversia de la ubicación óptima de un PICC. Algunos estudios consideran la vena cava superior (VCS) como la preferida para su inserción (1), mientras que otros prefieren la aurícula derecha (2). En el trabajo de Barría y cols. no se presentaron complicaciones relacionadas con su ubicación. La localización en la aurícula derecha presenta mayor riesgo de taponamiento cardíaco (3), por lo que existen estudios que avalan la inserción del catéter en extremidades inferiores, considerado un método seguro y eficaz, aunque de igual forma podría conllevar riesgos inherentes a su utilización y otros infrecuentes, como daño neurológico e incluso la muerte (4).

El estudio tiene como objetivo identificar el mecanismo etiológico y las complicaciones asociadas a la malposición de un PICC.

\section{DESCRIPCIÓN DEL CASO CLÍNICO}

Nace prematuro de 28 semanas a través de cesárea por rotura de membranas y trabajo de parto, Apgar $6-7$, peso 1.040 gr. Se conecta a ventilación mecánica y se instalan accesos vasculares: catéter umbilical arterial y venoso y tras término de su utilización, además, se instala PICC en extremidad inferior derecha. La decisión de colocar un catéter por vía femoral correspondió a una decisión primaria.
Los requerimientos de oxígeno disminuyen progresivamente. Inicia nutrición parenteral (NPT). Al 5to día, presenta distención abdominal, ausencia de deposiciones, hiperglicemia, glucosuria, apneas e hipoactividad. Se sospecha cuadro infeccioso, por lo que inicia tratamiento antibiótico. Al 7mo día, se observa aumento de volumen en región inguinal derecha, induración, eritema, retención urinaria y disminución de los movimientos en extremidades inferiores e insuficiencia respiratoria global.

\section{METODOLOGÍA}

Los datos del caso se recabaron del Hospital Clínico Magallanes. En cuanto a los principios éticos, éstos se cumplieron cuidando la confidencialidad de la información obtenida.

El manejo del caso se contrarresta con la evidencia encontrada en buscadores como National Center for Biotechnology Information, U.S. National Library of Medicine y Scientific Electronic Library Online.

\section{RESULTADOS}

La tasa de colocación exitosa de un PICC es del 85\%-95\%. Es considerado un procedimiento seguro y eficaz que permite asegurar un acceso venoso central prolongado como lo fue en este caso. Tiene una menor tasa de complicaciones comparadas con otras líneas venosas centrales (5), sin embargo, existen complicaciones como la malposición que pueden ser mortales (6).

El primer informe del uso de un PICC para la infusión de NPT fue realizado por Shaw en 19737. El primer reporte de malposición ocurre en el año 1984, el paciente de nueve meses de edad falleció nueve días después. Una punción lumbar pesquisó la presencia de NPT en el espacio epidural (8). Desde entonces, se ha informado la colocación incorrecta del catéter en el plexo venoso paravertebral en varios documentos (9).

La malposición ocurre generalmente cuando el catéter se inserta a través de una extremidad inferior izquierda, sin embargo, también ser reportan en la extremidad derecha, pero en menor porcentaje (10), como ocurrió en este caso. El PICC se insertó en una vena no especificada de la extremidad inferior derecha, controlándose en un principio solo con radiografía anteroposterior, la cual no evidenciaba 
claramente una malposición. Posterior a la aparición de síntomas y deterioro respiratorio, se complementa con otros exámenes.

Las radiografías simples de tórax y abdomen (anteroposterior y lateral) mostraban el catéter desplazado hacia retroperitoneo (Fig. 1, 2 y 3). El manejo fue el retiro inmediato del PICC y la suspensión de NPT.

La ecografía abdominal del paciente muestra líquido laminal sobre psoas y engrosamiento parietal del músculo. Asímismo la ecografía de columna evidencia el espacio epidural ocupado por sustancia hiperecogénica con aplastamiento medular desde región lumbar hasta $\mathrm{C} 7$. Las punciones lumbares fueron todas hemorrágicas.

A menudo la malposición se descubre tardíamente posterior a la infusión de sustancias irritantes en los plexos paravertebrales (6).

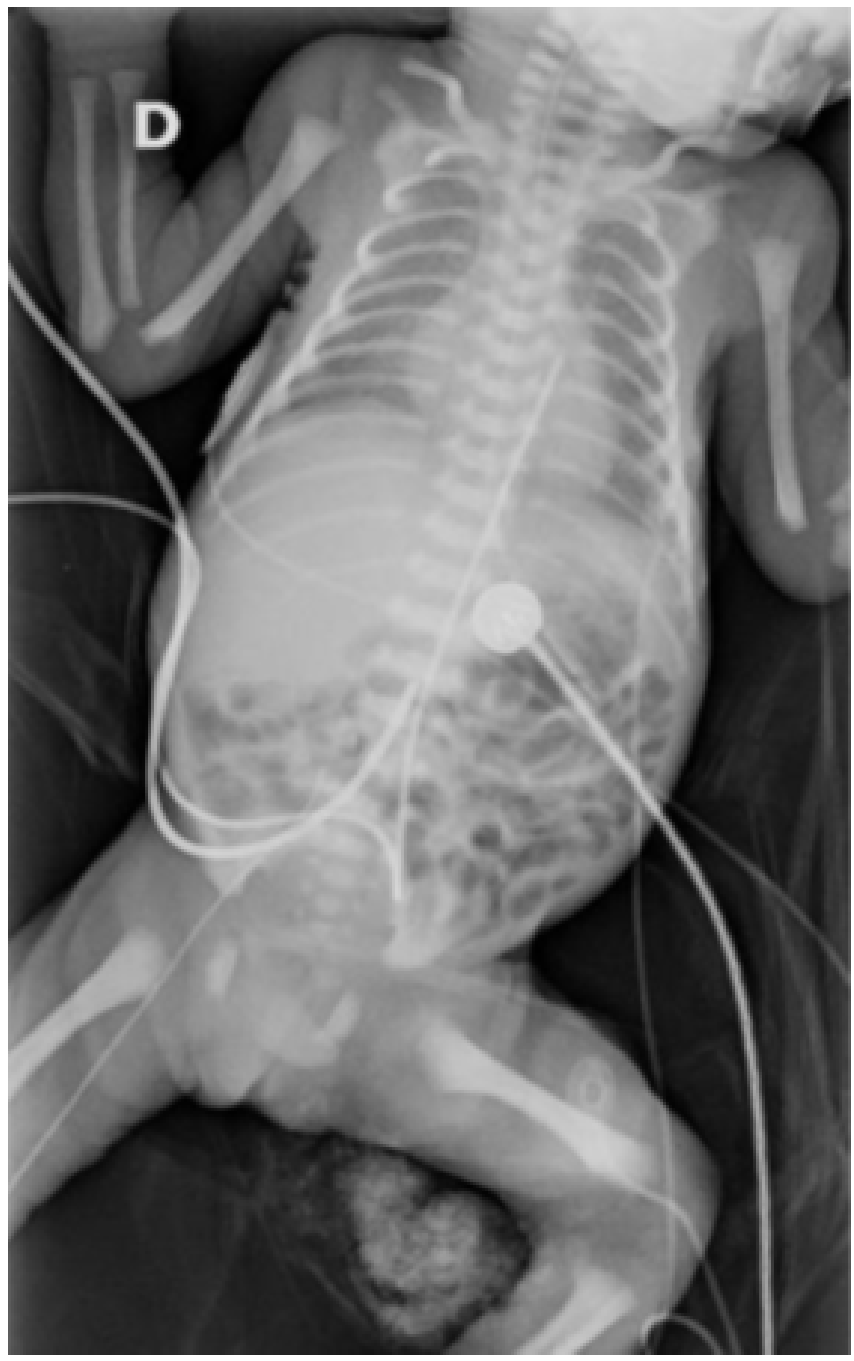

Figura 1. Radiografía antero-posterior en la que se observa el PICC instalado en la extremidad inferior derecha (imagen facilitada por Hospital Clínico Magallanes).



Figura 2. Radiografía antero-posterior del PICC en extremidad inferior derecha (imagen facilita-da por Hospital Clínico Magallanes).

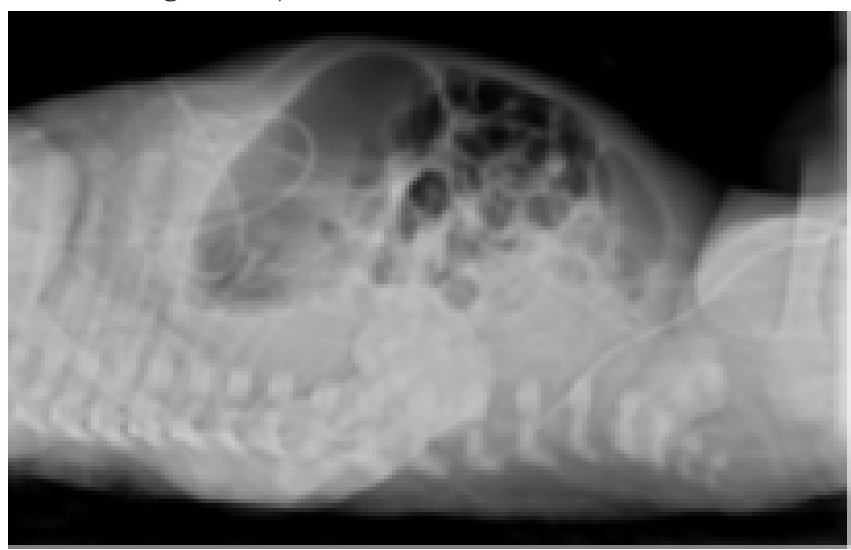

Figura 3. Radiografía lateral que evidencia el CVCP desplazado hacia retroperitoneo (imagen facilitada por Hospital Clínico Magallanes).

El hallazgo de NPT en punciones lumbares se pesquisó anteriormente en 7 infantes, de los cuales, 4 tenían menos de 32 semanas de edad gestacional y pesaban menos de 1.500 gr, al igual que el caso del presente estudio. La mayoría de los pacientes mostraron síntomas inespecíficos como sepsis, 
y aquellos con muy bajo peso al nacer, presentaron síntomas neurológicos (8) (tabla 1). Posterior a esto, se informaron 2 casos de recién nacidos extremadamente prematuros, que, sumado a los anteriores, 7 mostraron una recuperación completa sin secuelas aparentes, 1 desarrolló leucomalacia periventricular leve y otro falleció (4).

Los principales signos neurológicos que sugieren una posición inadecuada del PICC, incluyen: convulsiones, hipotonía, letargo, tetra o paraplejia y cuadriplejia flácida. También se pueden síndromes de sepsis e incluso la muerte. Estos aparecen generalmente desde el primer día hasta los 20 - 21 días posterior al procedimiento (6). Es este caso el paciente presentó signos localizados desde el 5 to día de vida, como aumento de volumen en región inguinal derecha, induración, eritema y distención abdominal. Asimismo, algunos signos inespecíficos como hiperglicemia, glucosuria, hipoactividad y apneas que se manejaron como sospecha de sepsis. Presentó síntomas neurológicos como retención urinaria, ausencia de deposiciones y disminución de los movimientos en extremidades inferiores. Además del deterioro respiratorio progresivo, que obligó a reconectarlo a ventilación mecánica. El paciente de 28 semanas sufrió una lesión e infarto medular a nivel de C7, paraplejia, tetraparesia flácida e insuficiencia respiratoria global que lo llevó a una traqueostomía entre los 91 y 144 días de vida. El paciente fallece a los 2 años y 2 meses por paro cardiorrespiratorio secundario a obstrucción de la vía aérea, sin posibilidad de ventilar.

De los 18 casos informados con PICC mal posicionados hasta ahora (8), solo 1 ha involucrado la inserción del lado derecho (6).

La vena iliaca común izquierda es más larga y oblicua en dirección, mientras que la vena iliaca común derecha es más corta y casi vertical (Fig. 4). El ángulo formado por la unión de la vena lumbar ascendente (VLA) con la vena iliaca común en el lado izquierdo es menos agudo y, en consecuencia, más propenso al paso inadecuado de un catéter ascendente hacia los plexos paraverbales, lo mismo podría pasar en el lado derecho, pero en menor porcentaje (11).

La infusión de NPT puede causar un flujo retrógrado de solución hiperosmolar en las venas de los plexos venosos vertebrales (Fig. 5). Debido a que cada VLA se comunica con venas epidurales en el canal espinal a través de venas intervertebrales, la infusión de NPT podría causar daño endotelial y la rotura de los vasos, liberando la solución al espacio epidural. Alternativamente, el catéter puede producir una perforación directa de la vena con entrada del fluido al espacio epidural (6) o al saco dural, pasando directamente al espacio subaracnoideo (12).

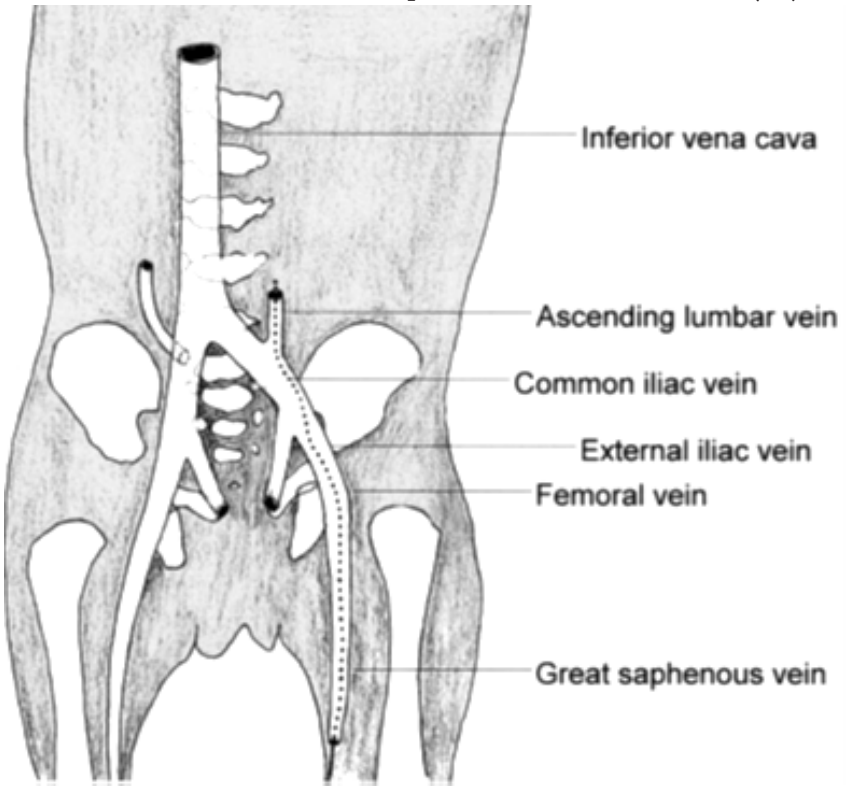

Figura 4. Imagen esquemática que muestra el camino inadecuado de un PICC (línea punteada) (4).

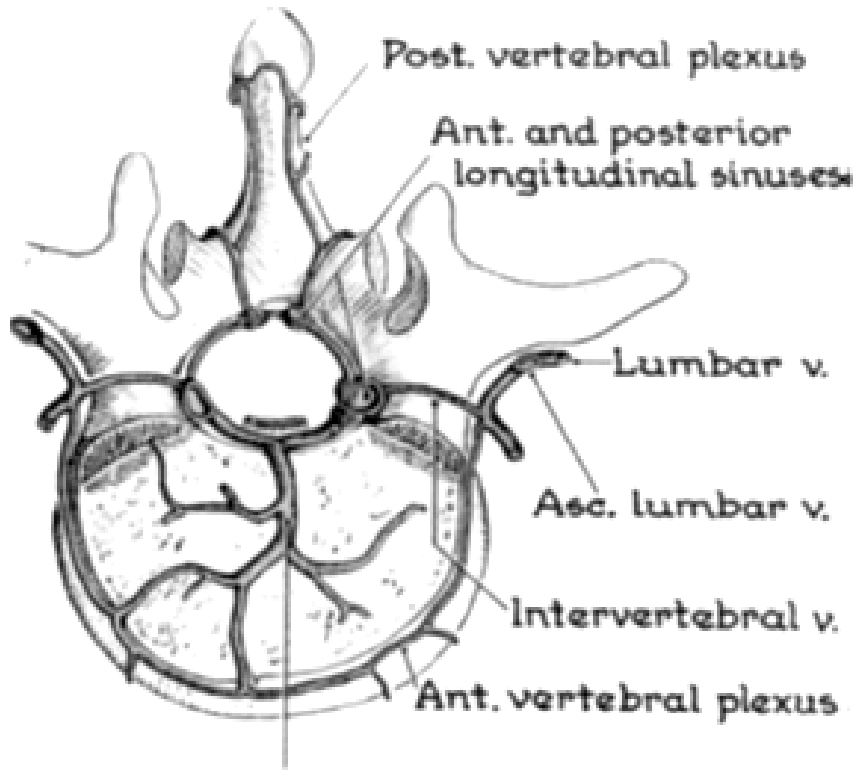

\section{Basivertebral vein}

Figura 5. Plexos venosos paravertebrales (13).

Un estudio de Chedid y cols., informa algunos signos sugerentes de malposición en radiografías simples. Describe 3 casos de recién nacidos prematuros con catéteres insertados en una vena safena izquierda, que terminaron en los plexos paraverte- 
brales. Las radiografías mostraron un signo en zigzag y un rizo en el área inguinal izquierda. En todos los casos la ubicación fue corroborada por estudios de contraste (14) (Fig. 6, 7 y 8).

En este caso, la radiografía anteroposterior evidencia el signo en zigzag que sugiere malposición y la radiografía lateral lo corrobora, sin embargo, no se realizaron estudios de contraste debido al riesgo de su infusión en el espacio epidural o subaracnoideo luego de aparecidos los síntomas.

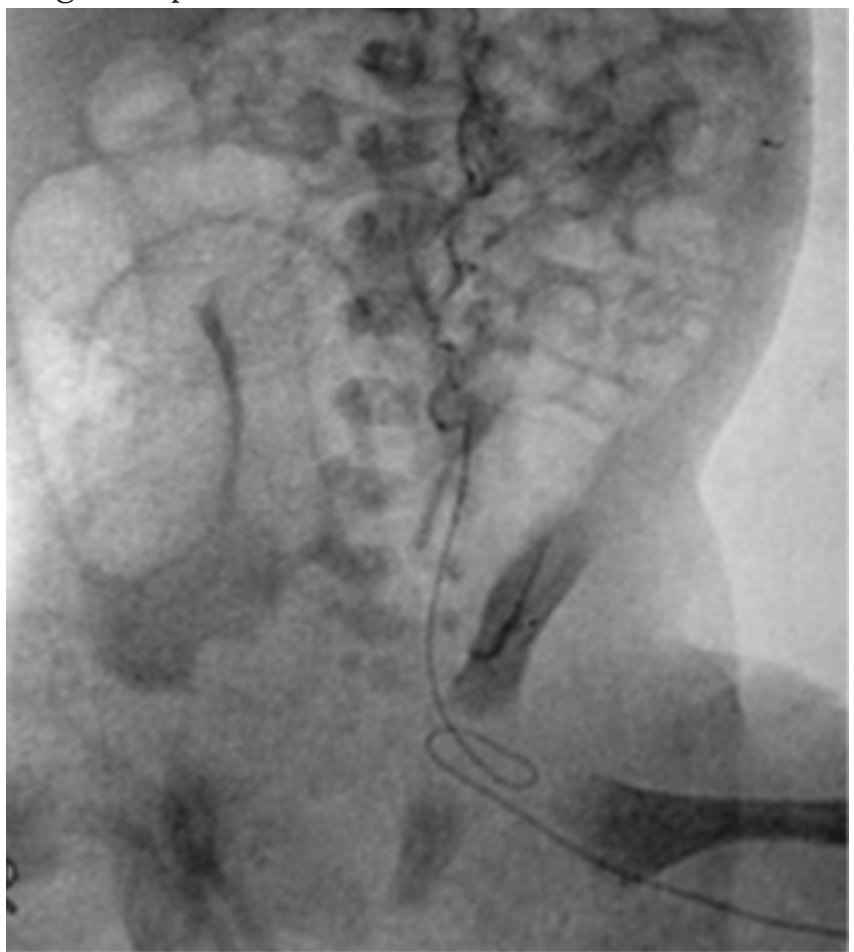

Figura 6. Caso 1. Imagen fluoroscópica de contraste que muestra un rizo inguinal izquierdo. La punta del PICC está a nivel de la L3. El medio de contraste muestra el plexo venoso lumbar ascendente (14)

\section{DISCUSIÓN}

Debido al alto riesgo de daño tisular causado por un volumen significativo de soluciones hiperosmóticas, es fundamental la identificación de los catéteres mal posicionados para la correcta función de éstos y la prevención de graves complicaciones (15).

Autores sugieren evitar el uso de la extremidad inferior izquierda para la inserción de un PICC debido al riesgo de maposición (14). Wrightson no encontró diferencias en las complicaciones entre las extremidades superiores e inferiores. En su opinión, la elección del sitio de inserción en los recién nacidos depende de la calidad de las venas disponibles y de la preferencia y habilidad del insertador (16).



Figura 7. Caso 2. Imagen de estudio de contraste que muestra un rizo inguinal izquierdo. La punta del PICC está a nivel de la tercera vértebra lumbar y puede observarse el plexo venoso lumbar ascendente (14).

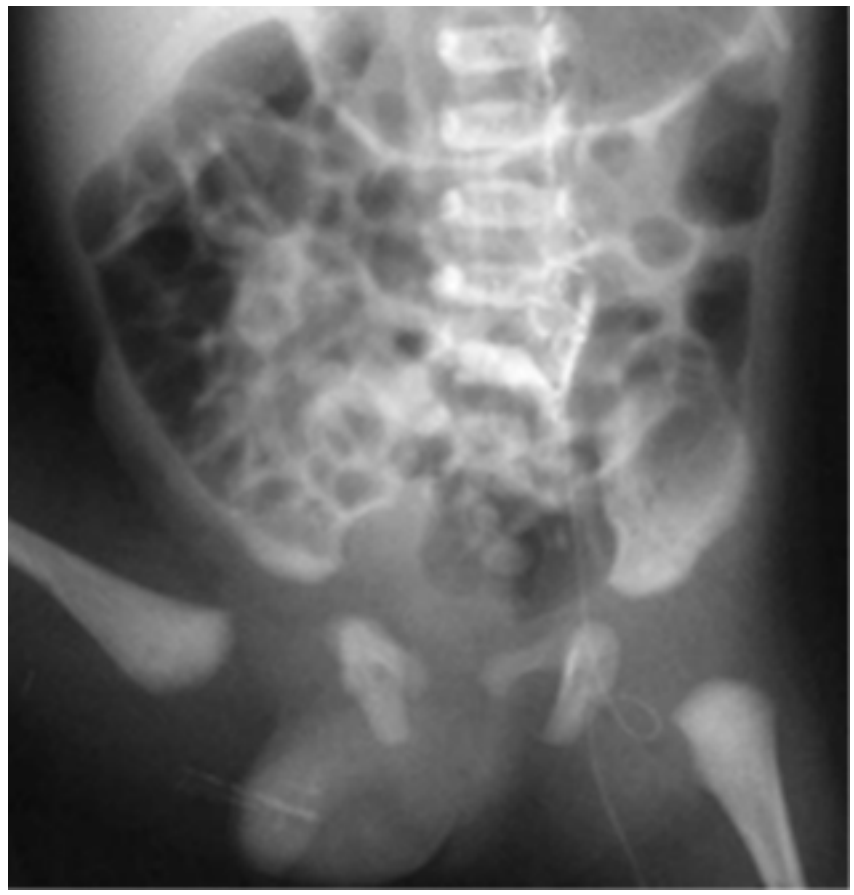

Figura 8. Caso 3. El PICC está curvado en el área inguinal izquierda. La punta del catéter está a nivel del tercer disco intervertebral. El plexo venoso lumbar ascendente se visualiza claramente (14) 
Hoang y cols., también comparó las tasas de complicaciones y concluyó que los catéteres insertados en la extremidad inferior, tuvieron tasas más bajas de infecciones, más tiempo de permanencia hasta la primera complicación y menor incidencia de colestasis a pesar de un mayor tiempo infusión de NPT. En este estudio, se sugiere que cuando sea posible, se debe usar catéteres insertados en las extremidades inferiores para la administración de NPT (17).

Debido a que la radiografía antero-posterior por sí sola no es suficiente para la localización de un catéter mal posicionado, la radiografía lateral, el estudio de contraste en casos seleccionados (Fig. 10) y la ecografía Doppler, son estudios que permiten verificar la posición del PICC y evitar las complicaciones potencialmente mortales (4).

En este caso, una radiografía simple lateral podría haber mostrado la desviación hacia retroperitoneo a nivel de la L4-L5, evitando la entrada del fluido en el espacio epidural y las consecuencias antes mencionadas.

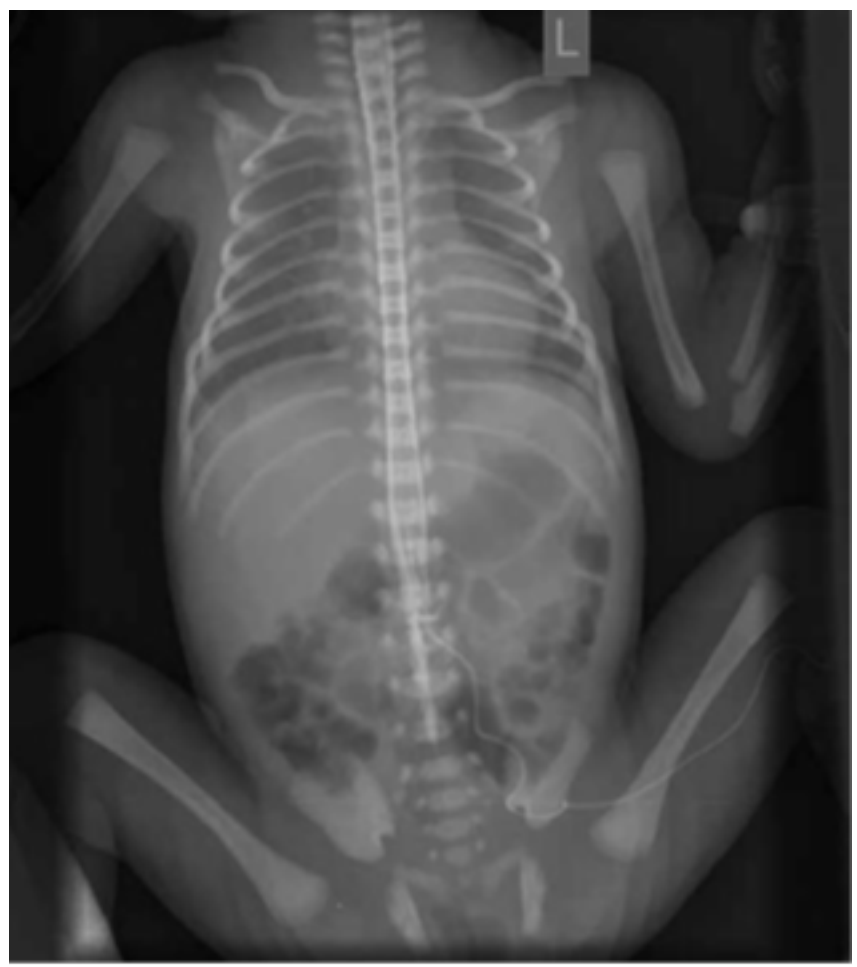

Figura 10. Radiografía que muestra el medio de contraste resaltando en el espacio epidural (4).
Observaciones para la práctica: Es importante tener presente que incluso los catéteres bien ubicados pueden migrar posteriormente a una posición anormal. Es por lo anterior, que nuestro rol de matronería es fundamental en la supervisión, control, pesquisa y derivación oportuna de cualquier signo de alteración o complicación asociada, debido a que esto permitirá la seguridad y eficacia de los procedimientos realizados en nuestros pacientes más complejos, además de la compresión del recién nacido como un ser íntegro e indefenso que precisa de nuestra habilidad como observadores y actores ante las situaciones más desafiantes.

\section{REFERENCIAS}

1. Beardsall K, White D, Pinto E, Kelsall A. Pericardial effusion and cardiac tamponade as complications of neonatal long lines: are they really a problem? Arch Dis Child Fetal Neonatal. 2003; 88(4): 292-295.

2. Cartwright D. Placement of neonatal central venous catheter tips: is the right atrium so dangerous? Arch Dis Child Fetal Neona-tal. 2002; 87(2): 155-156.

3. Barría M, Sanyander G. Cateterismo venoso central de inserción periférica en recién nacidos de cuidado intensivo. Rev Chil Pedi-atr. 2006; 77(2): 139146.

4. Clarke P, Wadhawan R, Smyth J, Emmerson A. Parenteral nutrition solution retrieved by lumbar puncture following left saphenous vein catheterization. Journal of paediatrics and child health. 2003; 39(5), 386-389.

5. Lussky R, Cifuentes R, Trower N. Paraspinal misplacement of percutaneously inserted central venous catheters. Pediatric radiology. 2006; 36(5): 466-467.

6. Zenker M, Rupprecht T, Hofbeck M, et al. Paravertebral and intraspinal malposition of transfemoral central catheters in newborns. J Paediatr. 2000; 136(6): 837-840.

7. Shaw J. Parenteral nutrition in the management of sick low birthweight infants. Pediatr Clin North Am. 1973; 20(2): 333-358.

8. Kelly M, Finer N, Dunbar L. Fatal neurologic complication of parenteral feeding through a central vein catheter. Am K Dis Child. 1984; 138(4): 352-353.

9. Chen C, Tsao P, Yau K. Paraplegia: complication of percutaneous central venous line malposition. Pediatr Neurol. 2001; 24(1): 65-68. 
10. Lavandosky G, Gomez R, Montes J. Potentially lethal misplacement of femoral central venous catheters. Crit Care Med. 1996; 24(5): 893-896.

11. Miller M, Handel S, Coan J. Transfemoral lumbar epidural venography. Am J Roentgenol. 1976; 126(5): 1003-1009.

12. White L, Montes J, Chaves-Carballo E, Presberg $\mathrm{H}$, Young $\mathrm{L}$. Radiological case of the month. Subarachnoid malposition of a femoral vein catheter. Am J Dis Child. 1987; 141(8): 903-904.

13. Williams P, Gray's Anatomy, British edn, 38th edn. Churchill Livingstone, New York, 1995.

14. Chedid F, Abbas A, Morris L. Radiographic inguinal curl may indicate paraspinal misplacement of percutaneously inserted central venous catheters: report of three cases. Pediatr Radiol. 2005; 35(7): 684-687.

15. Pettit J. Neurologic Complications Resulting from malpositioned or malfunctioning central venous catheters. Newborn infant nurs rev. 2006; 6(4): 212-224.

16. Wrightson D. Peripherally inserted central catheter complications in neonates with upper versus lower extremity insertion sites. Adv Neonatal Care. 2013; 13(3): 198-204.

17. Hoang V, Sills J, Chandler M, Busalani E, Clifton-Koeppel R, Modanlou H. Percutaneously inserted central catheter for total parenteral nutrition in neonates: complications rates related to upper versus lower extremity insertion. Pediatrics. 2008; 121(5): 1152-1159.
Tabla 1. Infantes descritos previamente con solución de nutrición parenteral obtenida mediante punción lumbar (4).

\begin{tabular}{|c|c|c|c|c|c|}
\hline Autor & $\begin{array}{c}\text { Edad } \\
\text { paciente }\end{array}$ & $\begin{array}{c}\text { Sitio de } \\
\text { inserción }\end{array}$ & $\begin{array}{c}\text { Tiempo } \\
\text { entre } \\
\text { cateri- } \\
\text { zación y } \\
\text { síntomas }\end{array}$ & Signos & Desenlace \\
\hline $\begin{array}{c}\text { Kelly y } \\
\text { cols., } \\
1984\end{array}$ & $\begin{array}{l}\text { RNT, } 9 \\
\text { meses }\end{array}$ & $\begin{array}{c}\text { Vena } \\
\text { safena } \\
\text { izquierda }\end{array}$ & 9 días & $\begin{array}{c}\text { Pirexia, } \\
\text { taquipnea, } \\
\text { mala perfu- } \\
\text { sión, acido- } \\
\text { sis metabó- } \\
\text { lica }\end{array}$ & Muerte \\
\hline $\begin{array}{c}\text { Mah y } \\
\text { cols., } \\
1991 \\
\end{array}$ & $\begin{array}{c}\text { RNT, } 12 \\
\text { días }\end{array}$ & $\begin{array}{c}\text { Vena } \\
\text { femoral } \\
\text { izquierda }\end{array}$ & 11 días & $\begin{array}{c}\text { Mala perfu- } \\
\text { sión, palidez, } \\
\text { taquipnea }\end{array}$ & $\begin{array}{c}\text { Recupe- } \\
\text { ración } \\
\text { total }\end{array}$ \\
\hline $\begin{array}{c}\text { Odai- } \\
\text { bo y } \\
\text { cols., } \\
1992\end{array}$ & $\begin{array}{c}\text { RNT, } 15 \\
\text { días }\end{array}$ & $\begin{array}{c}\text { Vena } \\
\text { safena } \\
\text { izquierda }\end{array}$ & 7 días & $\begin{array}{l}\text { Pirexia, piel } \\
\text { reticulada, } \\
\text { mala perfu- } \\
\text { sión }\end{array}$ & $\begin{array}{c}\text { Recupe- } \\
\text { ración } \\
\text { total }\end{array}$ \\
\hline $\begin{array}{c}\text { Bass y } \\
\text { Lewis, } \\
1995\end{array}$ & $\begin{array}{l}\text { RNPT } 27 \\
\text { sema- } \\
\text { nas, } 27 \\
\text { días }\end{array}$ & $\begin{array}{c}\text { Vena } \\
\text { safena } \\
\text { izquierda }\end{array}$ & 10 días & $\begin{array}{c}\uparrow \text { distrés } \\
\text { respirato- } \\
\text { rio, } \uparrow \mathrm{FiO}_{2} \text {, } \\
\text { letargia, } \\
\text { hipotonía, } \\
\text { convulsiones } \\
\text { mioclónicas }\end{array}$ & $\begin{array}{c}\text { Retraso } \\
\text { motor y } \\
\text { cognitivo } \\
\text { modera- } \\
\text { do }\end{array}$ \\
\hline Rajan y & $\begin{array}{l}\text { RNPT } \\
28 \text { se- } \\
\text { manas, } \\
14 \text { días }\end{array}$ & $\begin{array}{c}\text { Vena } \\
\text { safena } \\
\text { izquierda }\end{array}$ & 7 días & $\begin{array}{c}\text { Convulsio- } \\
\text { nes tónico- } \\
\text { clónicas } \\
\text { agudas }\end{array}$ & $\begin{array}{c}\text { Recupe- } \\
\text { ración } \\
\text { total }\end{array}$ \\
\hline $\begin{array}{l}\text { Berg- } \\
\text { man y } \\
\text { cols., } \\
1999\end{array}$ & $\begin{array}{c}\text { RNPT } 31 \\
\text { sema- } \\
\text { nas, } 7 \\
\text { días }\end{array}$ & $\begin{array}{c}\text { Vena } \\
\text { safena } \\
\text { izquierda }\end{array}$ & 4 días & $\begin{array}{c}\text { } \text { apoyo } \\
\text { ventilatorio, } \\
\text { letargia, } \\
\text { hipotonía, } \\
\text { mala perfu- } \\
\text { sión } \\
\end{array}$ & $\begin{array}{c}\text { Recupe- } \\
\text { ración } \\
\text { total }\end{array}$ \\
\hline $\begin{array}{l}\text { Vid- } \\
\text { wans } \\
\text { y cols., } \\
2000\end{array}$ & $\begin{array}{l}\text { RNPT } 27 \\
\text { sema- } \\
\text { nas, } 13 \\
\text { días }\end{array}$ & $\begin{array}{c}\text { Vena } \\
\text { safena } \\
\text { izquierda }\end{array}$ & 2 días & $\begin{array}{c}\uparrow a p n e a s, \\
\text { acidosis } \\
\text { respirato- } \\
\text { ria, palidez, } \\
\text { letargia }\end{array}$ & $\begin{array}{c}\text { Recupe- } \\
\text { ración } \\
\text { total }\end{array}$ \\
\hline
\end{tabular}

